\title{
Erratum: Global phylogeography and evolutionary history of Shigella dysenteriae type 1
}

Elisabeth Njamkepo, Nizar Fawal, Alicia Tran-Dien, Jane Hawkey, Nancy Strockbine, Claire Jenkins, Kaisar A. Talukder, Raymond Bercion, Konstantin Kuleshov, Renáta Kolínská, Julie E. Russell, Lidia Kaftyreva, Marie Accou-Demartin, Andreas Karas, Olivier Vandenberg, Alison E. Mather, Carl J. Mason, Andrew J. Page, Thandavarayan Ramamurthy, Chantal Bizet, Andrzej Gamian, Isabelle Carle, Amy Gassama Sow, Christiane Bouchier, Astrid Louise Wester, Monique Lejay-Collin, Marie-Christine Fonkoua, Simon Le Hello, Martin J. Blaser, Cecilia Jernberg, Corinne Ruckly, Audrey Mérens, Anne-Laure Page, Martin Aslett, Peter Roggentin, Angelika Fruth, Erick Denamur, Malabi Venkatesan, Hervé Bercovier, Ladaporn Bodhidatta, Chien-Shun Chiou, Dominique Clermont, Bianca Colonna, Svetlana Egorova, Gururaja P. Pazhani, Analia V. Ezernitchi, Ghislaine Guigon, Simon R. Harris, Hidemasa Izumiya, Agnieszka Korzeniowska-Kowal, Anna Lutyńska, Malika Gouali, Francine Grimont, Céline Langendorf, Monika Marejková, Lorea A.M. Peterson, Guillermo Perez-Perez, Antoinette Ngandjio, Alexander Podkolzin, Erika Souche, Mariia Makarova, German A. Shipulin, Changyun Ye, Helena Žemličková, Mária Herpay, Patrick A. D. Grimont, Julian Parkhill, Philippe Sansonetti, Kathryn E. Holt, Sylvain Brisse, Nicholas R. Thomson and François-Xavier Weill

Nature Microbiology 1, 16027 (2016); published 21 March 2016; corrected 3 October 2016

In the original version of this Letter, co-author Simon Le Hello's name was coded wrongly resulting in it being incorrect when exported to citation databases. This has now been corrected, though no visible changes will be apparent. 